\title{
Gamut Extension for Cinema: Psychophysical Evaluation of the State of the Art, and a New Algorithm
}

\author{
Syed Waqas Zamir, Javier Vazquez-Corral, and Marcelo Bertalmío \\ Department of Information and Communication Technologies, \\ Universitat Pompeu Fabra, Barcelona, Spain
}

\begin{abstract}
Wide gamut digital display technology, in order to show its full potential in terms of colors, is creating an opportunity to develop gamut extension algorithms (GEAs). To this end, in this work we present two contributions. First we report a psychophysical evaluation of GEAs specifically for cinema using a digital cinema projector under cinematic (low ambient light) conditions; to the best of our knowledge this is the first evaluation of this kind reported in the literature. Second, we propose a new GEA by introducing simple but key modifications to the algorithm of Zamir et al. ${ }^{1}$ This new algorithm performs well in terms of skin tones and memory colors, with results that look natural and which are free from artifacts.
\end{abstract}

Keywords: Gamut Mapping Algorithms, Gamut Extension for Cinema, Psychophysical Evaluation

\section{INTRODUCTION}

The term "color gamut" refers to the set of colors that a display device is able to reproduce. Modern cameras are capable of capturing wide color gamuts on their sensors and these gamuts are different (usually larger) than the standards used for the distribution of content such as ITU-R (International Telecommunication Union-Radiocommunication) Rec. 709 for broadcast industry and DCI (Digital Cinema Initiatives) P3 for cinema industry. Therefore, the input content needs to be reproduced with respect to these standard gamuts, and the process that transforms colors from an input to an output gamut is called Gamut Mapping (GM). The fundamental goal of GM is to reproduce input material in accordance with the color characteristics of a display device such that the reproduction stays perceptually faithful to the original material. The intent of creating a perceptual gamut mapped reproduction is a challenging task, mainly because of the fact that the color perception is a complicated phenomenon and far from being fully understood. ${ }^{2}$

Modern image acquisition technology has a potential to capture movies that have a much wider range of colors than regular display devices (digital cinema projector, HD TV, etc.) have. Therefore, in the film industry an important problem at the post production stage is to adapt colors for different display devices such that a person watching a movie on any of these screens perceives the same colors. ${ }^{3}$ To reach this objective, colorists, by using 3D Look Up Tables (LUTs), map the colors of the movie material into the reduced gamut of each type of target device. This semi-automated process is difficult, time consuming and prone to human errors, and therefore it makes an automated gamut reduction (GR) very desirable.

On the other hand, state of the art digital cinema projectors have a wide color gamut, but often the movies arriving at these new projectors have had their gamuts reduced so as to prevent issues with old or regular projectors (that have a smaller gamut). Therefore, in order to make use of the full potential of new projectors in terms of color, a process called gamut extension (GE) is needed, transforming colors from a smaller source gamut to a larger destination gamut. With the introduction of lasers in digital display technology ${ }^{4}$ it is foreseeable that in a few years the gamut of a standard digital cinema projector will be increased to a dramatic extent, often surpassing the color gamut capabilities of cameras.

One of the major problems in GM is the evaluation of gamut mapping algorithms (GMAs). There are two possible ways of evaluating GMAs either subjectively or objectively. In the case of subjective evaluation, subjects in psychophysical experiments are asked to choose or rate the reproductions based on a criterion (preference or accuracy). Most psychophysical studies to evaluate gamut mapping closely follow the guidelines of the International Commission on Illumination (CIE) ${ }^{5}$ in which the conditions of experimental set-ups are described. It is certainly a difficult task to declare whether a GMA is better than all the previous algorithms, and the reason is that the degree of accuracy changes with the change in conditions: what is often reported ${ }^{2}$ is that an algorithm that performs well for a few combinations of source and destination gamuts shows

\{waqas.zamir, javier.vazquez, marcelo.bertalmio\} @upf.edu 
poor performance for some other combinations, and many algorithms are image dependent, meaning that they only work efficiently for some images.

Subjective evaluation is time consuming, expensive and requires different observers and repeated trials in order to provide reliable results. The alternative is to use error metrics that estimate the color quality of the image reproduction. This objective evaluation makes use of some color fidelity metric that aims at predicting how faithful is the reproduction as compared with the original material.

GE has received much less attention than gamut reduction as we shall see in the next section. Therefore, as our first contribution we present the results of an evaluation which is, to the best of our knowledge, the first psychophysical evaluation of GEAs done specifically for cinema, using a digital cinema projector in cinematic (low ambient light) conditions. Also, we compare the results of the psychophysical experiment with those of current metrics for gamut mapping and color fidelity in images in order to identify the best performing image quality measure for the GE problem.

As our second contribution we propose a new GEA based on the work of Zamir et al., ${ }^{1}$ introducing simple but key modifications that eliminate the problems of saturation and over-enhancement of contrast that can be seen in the results obtained with that method.

\section{RELATED WORK}

GMAs that are dedicated to perform gamut reduction, to which we refer as gamut reduction algorithms (GRAs), are classified into two broad categories: global GRAs and local GRAs. The global GRAs (also called non-local or non-adaptive) are further classified into two sub-classes: clipping and compression. Gamut clipping is the simplest approach to perform gamut mapping where the colors that fall inside the destination gamut are left unmodified while those that are outside are projected onto the destination gamut boundary by following a mapping criterion. The works in literature that make use of clipping are described in. ${ }^{6,7}$ All gamut clipping methods project whole out-of-gamut color segments to single points on the destination gamut boundary, and this process may produce gamut mapped images with visible loss of color gradient and texture. To overcome this issue, gamut compression algorithms treat all the colors present in an input image and map its gamut to the destination gamut either linearly or non-linearly. Some widely used gamut compression algorithms are proposed in. ${ }^{8,9}$ Gamut compression algorithms map a larger out-of-gamut color segment to a smaller in-gamut color segment which may cause significant loss in saturation, especially when the difference between source and target gamut is large.

Local GRAs are adaptive and take into account the spatial color information of the original image while fitting the color gamut of an image into the gamut of a given device. Meyer and Barth ${ }^{10}$ proposed a spatial GM algorithm where the lightness of the source image is first compressed in Fourier domain using a low pass filter followed by the chroma compression along lines of constant hue and (mapped) lightness. Then, the high frequency detail of the image is added back to the gamut compressed image. Bala et al. ${ }^{11}$ described a similar method where the source image is processed using a gamut clipping algorithm, and as a second step the difference of luminance between the original and the mapped image is calculated. Subsequently, a spatial filter is applied to the difference and the result is added back to the gamut mapped image. In the algorithms of Meyer and Barth ${ }^{10}$ and Bala et al., ${ }^{11}$ another stage of gamut clipping is integrated to process the resulting image in case the spatial filtering operation places a few pixels outside the destination gamut. Morovič and Wang ${ }^{12}$ introduced a multi-level, full color GMA that first decomposes the image into a number of spatial frequency bands. Secondly, at the lowest frequency band, the lightness compression is applied followed by the application of initial gamut mapping. Then, the next higher frequency band is added to the gamut mapped image and again gamut mapping is applied to the resulting image. This step is repeated until the highest frequency band is reached. The core idea was to transform the gamut of the original image into the destination gamut while preserving the original image's overall information into the reproduction. Kimmel et al. ${ }^{13}$ presented a variational approach where the GM problem is formulated as a quadratic programming optimization form, which is guaranteed to have a unique optimal solution provided that the target device has a convex gamut. They have defined a functional to measure both the image difference and its derivatives to perform GM. McCann ${ }^{14}$ proposed a Retinex-inspired ${ }^{15}$ framework that performs spatial comparisons to preserve the local gradients to obtain the final gamut mapped image. A similar multi-resolution GM approach that adapts the original image is suggested by Farup et al. ${ }^{16}$ Zolliker and Simon ${ }^{17}$ presented an unsharp masking scheme that combines the spatial methods with the global GM to retain the local contrast information in the reproduction. Alsam and Farup ${ }^{18}$ proposed an iterative GRA that at iteration level zero behaves as a gamut clipping algorithm, whereas, by increasing the number of iterations, the solution approaches spatial gamut mapping. Zamir et al. ${ }^{19}$ proposed a spatial GRA that performs GM by reducing the contrast using 
a perceptual image energy functional. ${ }^{20}$ Later, this work was extended by Vazquez-Corral et al. ${ }^{21}$ to better preserve the saliency of the original image in the reproduced one. Nakauchi et al. ${ }^{22}$ define gamut mapping as an optimization problem where they use a perceptual metric to minimize the perceived differences between the original and the reproduced image in order to obtain the final gamut mapped image. There are some problems associated with local GRAs, such as that these methods are often computationally expensive, or based on many assumptions, and may report halo artifacts.

The case of gamut extension is different from that of gamut reduction: only a handful of gamut extension algorithms (GEAs) exist in the literature. One could think of simply taking any GRA and use the one-to-one mapping in the reverse direction to perform gamut extension, as Morovič comments in. ${ }^{2}$ However, the key struggle is to produce gamut extended images that look natural, pleasant and perceptually close to the original images.

Similar to GRAs, gamut extension algorithms also have two categories: global GEAs and local GEAs. Hoshino ${ }^{23}$ proposed the first global GEA that maps the colors of printed images to the color gamut of high-definition TV using a nonlinear tone reproduction curve on the lightness, and then the chroma is mapped along the lines of constant lightness and hue. However, the results were lacking naturalness and therefore a revised version of GEA was presented in. ${ }^{24}$ Kang et al. ${ }^{25}$ presented a GEA that was based on numerical fitting of subjective experimental data that was collected by allowing a group of observers to linearly (manually) extend lightness and chroma. Anderson et al. ${ }^{26}$ introduced a user assisted method where an expert was asked to expand the gamut of some key frames and the rest of the frames were corrected accordingly. Lui et al. ${ }^{27}$ presented a gamut expansion method in the CIELuv color space that extends from an anchor point while respecting the hue lines. Kim et al. ${ }^{28}$ described a GEA with three type of extension strategies: chroma mapping, mapping along lines from the origin (which they called vector mapping) and adaptive mapping that is a compromise between the first two strategies. Casella et al. ${ }^{29}$ introduced a sigmoid function into the linear mapping techniques so that it does not consider objects of low chroma equivalent to those of high chroma. Subjective evaluations of GMAs were performed in Mujis et al., ${ }^{30}$ and Laird et al. ${ }^{31}$ In the latter, they proposed GEAs that are explained in more details in the next section, and we use these algorithms to compare with the results of our GE algorithm. Heckaman et al. ${ }^{32}$ suggested an algorithm that exploits the use of memory colors such as the green of the grass or the blue of the sky while preserving the flesh tones.

One of the major drawbacks of global GEAs is that they often produce gamut mapped images that are highly saturated, artificial in appearance and off from the original images. Local GEAS are able to better address these issues. Li et al. ${ }^{33}$ proposed a multilevel GEA where first the global GEA is applied using a nonlinear hue-varying extension function and as a second step a human contrast sensitivity function is used in the local gamut mapping to avoid the over-contrast problem and to retain the local information in the final reproduced image. Zamir et al. ${ }^{1}$ presented a spatial GEA that enhances the contrast using the perceptual variational model ${ }^{20}$ leading to an expansion of the color gamut. Zamir et al. ${ }^{1}$ report that in the reproduction a few pixels tend to go towards black color if the input image has medium-to-large dynamic range. To deal with this issue, they have added a preprocessing stage that gives a shift to the histogram of each channel of the input image, from low towards high intensity values. The preprocessing stage enables the algorithm of Zamir et al. ${ }^{1}$ to generate color reproductions that are saturated, pleasing and natural in appearance, and perceptually more closer to the original image as compared with the Same-Drive-Signal algorithm, ${ }^{31}$ but it is still far from being the ideal solution to deal with the gamut extension problem.

As mentioned before, one can evaluate GMAs either subjectively, or by using color fidelity metrics. The most common subjective method is the pair comparison, where observers are asked to choose which of two different gamut-mapped versions of an image is more faithful to the original. Dugay et al ${ }^{34}$ present a psychophysical study where they evaluate five spatial and global GRAs. Other psychophysical studies that make use of the paired comparison paradigm to identify the best performing GRA are presented in ${ }^{35} .{ }^{36}$ Laird et al ${ }^{31}$ propose five global GEAs and conduct two psychophysical experiments using simulated and actual wide-gamut display to evaluate the efficiency of their algorithms.

In case of objective evaluation, many image quality metrics ${ }^{37-40}$ exist in the literature to quantify the results of GMAs; a few are perceptually-based, and others are pixel wise metrics. Lissner et al.$^{40}$ present a perceptually-based color image difference (CID) metric that particularly emphasises on the assessment of gamut-mapped images. The CID works quite efficiently for the GR problem. Hardeberg et al. ${ }^{41}$ and Baranczuk et al. ${ }^{39}$ identify by using psychophysical experiments the best performing objective metric for the GR problem, but the ranking of color metrics may not be consistent in the context of GE. And to the best of our knowledge there doesn't exist any study in the literature that aims to identify a color metric for the GE case. 


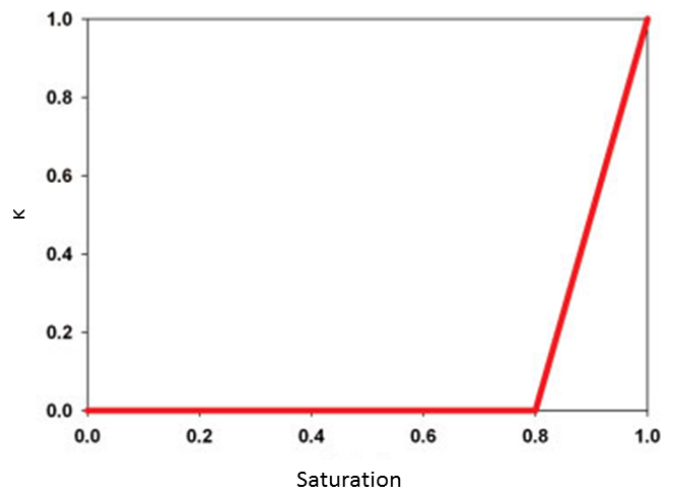

Figure 1: Mixing function for HCM. Figure is taken from. ${ }^{31}$

\section{PSYCHOPHYSICAL EVALUATION OF GAMUT EXTENSION ALGORITHMS FOR CINEMA}

In this section we describe four GE algorithms and also we present a new gamut extension algorithm.

\subsection{Same Drive Signal (SDS)}

The most commonly used GE algorithm is the SDS, ${ }^{31}$ where the RGB primaries of the input material are mapped to the RGB primaries of the display device enabling the SDS algorithm to make full use of the wide-gamut of the display.

\subsection{Hybrid Color Mapping (HCM)}

The true-color algorithm maintains the color information of the input image into the destination gamut without applying any sort of extension, meaning that the output of true-color is nothing but a representation of the input image in a wide gamut colorspace:

$$
\left[\begin{array}{l}
R \\
G \\
B
\end{array}\right]_{\text {true-color }}=M_{\text {Destination }}^{-1} M_{\text {Source }}\left[\begin{array}{c}
R \\
G \\
B
\end{array}\right]_{\text {Source }},
$$

where both $M_{\text {Source }}$ and $M_{\text {Destination }}$ are $3 \times 3$ transformation matrices to convert the values of RGB to XYZ colorspace.

The HCM algorithm ${ }^{31}$ linearly combines the output of the true-color and SDS algorithms based on the saturation of the input image:

$$
\left[\begin{array}{l}
R \\
G \\
B
\end{array}\right]_{H C M}=(1-\kappa)\left[\begin{array}{l}
R \\
G \\
B
\end{array}\right]_{\text {true-color }}+\kappa\left[\begin{array}{l}
R \\
G \\
B
\end{array}\right]_{S D S}
$$

where $\kappa$ is a mixing factor that works as a function of saturation as shown in figure 1.

The key idea behind the HCM algorithm is to preserve natural colors, leaving unmodified the low saturated colors such as skin tones, while mapping highly saturated colors using the SDS algorithm.

\subsection{Chroma Extension (CE)}

The SDS algorithm works by extending the input signal to the destination gamut. However, hue differences between source and destination gamut may lead SDS to produce an image that exhibits hue shifts. To tackle this problem, the chroma extension algorithm ${ }^{31}$ maps colors of the source gamut to the reproduction gamut along lines of the chroma axis in the CIELch colorspace, while keeping lightness and hue constant. 


\subsection{Lightness Chroma Adaptive (LCA)}

The lightness-chroma adaptive algorithm ${ }^{31}$ performs GE by altering both lightness and chroma while keeping the hue constant.

Both CE and LCA algorithms make use of the so called High Chroma Boost (HCB) function which smoothly maps colors of an input image in a manner that the high chroma objects get more boost in saturation than the low chroma ones. This approach aims to preserve memory color objects as they often have less saturated values, and extends colors of artificial objects (such as plastics) that have high chroma.

\subsection{Proposed GE Algorithm}

Zamir et al. ${ }^{1}$ proposed a spatial GEA that adapts the image energy functional of Bertalmío et al. ${ }^{20}$ to expand from a smaller source gamut to a larger destination gamut. Their work of ${ }^{20}$ is closely related to the Automatic Color Enhancement (ACE) model of Rizzi et al., ${ }^{42}$ and both the algorithms of ${ }^{20}$ and $^{42}$ mimic global and local perceptual properties of the human visual system and are very closely related to the Retinex theory of color vision. ${ }^{15,43}$ Zamir et al. ${ }^{1}$ presented an adapted image energy functional

$$
E(I)=\frac{\alpha}{2} \sum_{x}(I(x)-\mu)^{2}-\frac{\gamma}{2} \sum_{x} \sum_{y} w(x, y)|I(x)-I(y)|+\frac{\beta}{2} \sum_{x}\left(I(x)-I_{0}(x)\right)^{2},
$$

whose minimization extends the gamut of an input image. In eq. (3) $\alpha, \beta$ and $\gamma$ are constant and positive weights, $I$ is a color channel $(R, G$ or $B), \mu$ is the average value of each channel of original image $I_{0}, w(x, y)$ is a normalized Gaussian kernel of standard deviation $\sigma$, and $I(x)$ and $I(y)$ are two intensity levels at pixel locations $x$ and $y$ respectively.

By minimizing the image energy in Eq. (3) the aim is to maximize the contrast (second term of the functional), while not departing too much from the original image (third term) and also preserving the gray world hypothesis (first term). It is formulated in ${ }^{20}$ that the solution to the minimization of Eq. (3) can be found as the steady state of the evolution equation

$$
I^{k+1}(x)=\frac{I^{k}(x)+\Delta t\left(\alpha \mu+\beta I_{0}(x)+\frac{\gamma}{2} R_{I^{k}}(x)\right)}{1+\Delta t(\alpha+\beta)}
$$

where the initial condition is $I^{k=0}(x)=I_{0}(x)$. The function $R_{I^{k}}(x)$ indicates the contrast function:

$$
R_{I^{k}}(x)=\frac{\sum_{y \in \mathfrak{I}} w(x, y) s\left(I^{k}(x)-I^{k}(y)\right)}{\sum_{y \in \mathfrak{I}} w(x, y)}
$$

where $x$ is a fixed image pixel and $y$ varies across the image. The slope function $s()$ is a regularized approximation to the sign function, which appears as it is the derivative of the absolute value function in the second term of the functional; in ${ }^{20}$ they choose for $s()$ a polynomial of degree 7 .

The GEA of Zamir et al., ${ }^{1}$ due to its inherent behavior to expand colors by increasing the contrast of the image, produces results with over-enhanced contrast, which in turn makes a few colors to go towards black (loss of saturation) as it is visible in figure $2 \mathrm{~b}$. It can be seen that the overall contrast of the reproduction is increased to an unnatural extent, making it depart away from the original image perceptually. Also, the over-enhancement of contrast causes loss of color details as it is shown in the area highlighted by a bounding box in figure $2 \mathrm{~b}$. To overcome these problems, we propose a new GEA based on ${ }^{1}$ where we decouple the color information from the luminance of the RGB input image by converting it to CIELab colorspace, and apply the evolution Eq. (4) until steady state only on the color components 'a' and 'b' while keeping the luminance of the input image constant. We keep the sign of the contrast coefficient $\gamma$ positive in order to maximize the contrast that leads our algorithm to extend the color gamut of an image. Also we define the coefficient $\alpha$ as $\frac{|\gamma|}{20}$. To select the value of $\gamma$ that is adequate to map colors of the source image to the destination gamut, we pick the minimum $\gamma$ that makes the original gamut exceed the destination gamut upto a certain threshold level $\left(T_{e}\right)$. This threshold $T_{e}$ controls the level of saturation; a large value of $T_{e}$ indicates a higher saturation level, whereas a small value of $T_{e}$ yields a less saturated output. After this, the colors that were placed outside the destination gamut in the previous stage are mapped back inside using our gamut reduction algorithm.

We show an example in figure 2 where we map colors from the smaller 'Toy' gamut to the larger 'Rec. 709' gamut (gamuts are shown in figure 3, and primaries are mentioned in table 1). It can be seen that the reproductions obtained using 


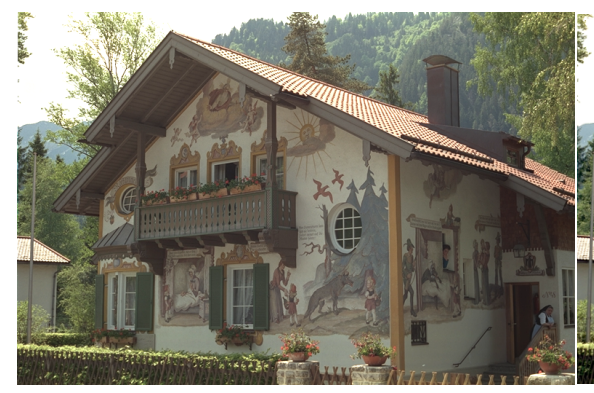

(a)

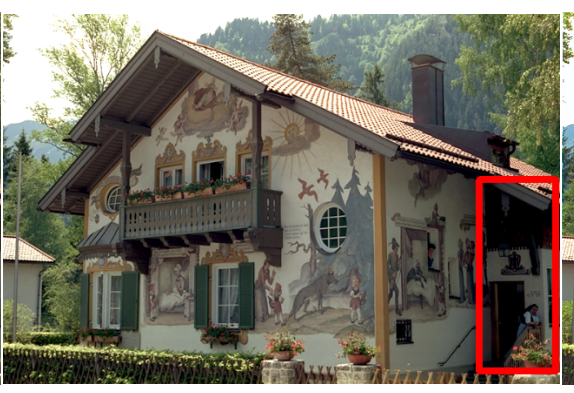

(b)

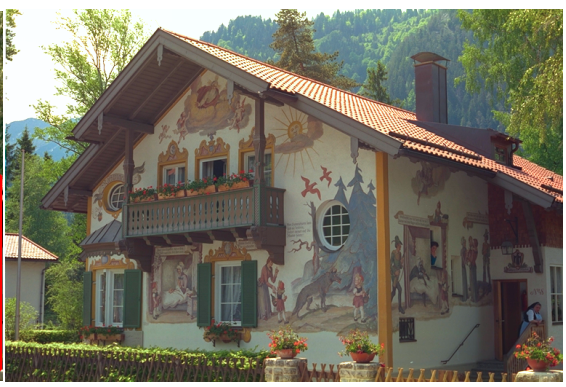

(c)

Figure 2: Gamut extension example. (a) Input image. (b) Result of Zamir et al. ${ }^{1}$ (c) Our reproduction. Original image is courtesy of Kodak.

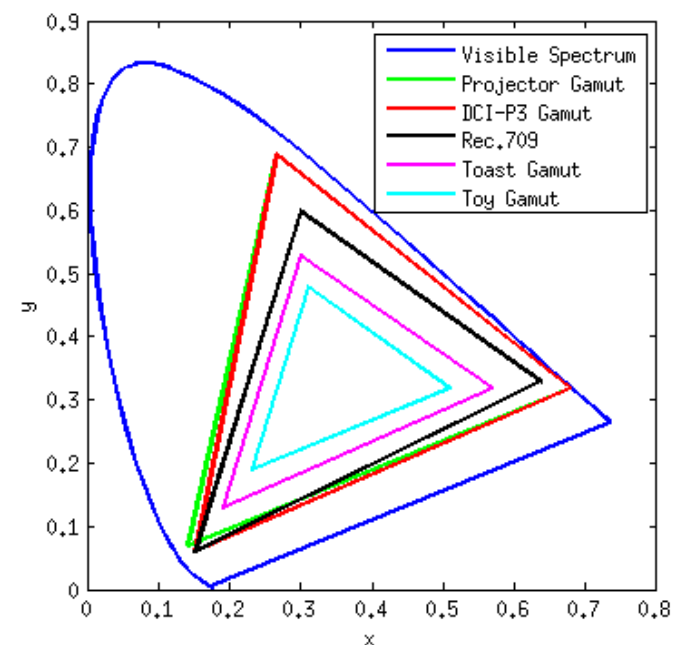

Figure 3: Gamuts on chromaticity diagram.

Table 1: Primaries of gamuts.

\begin{tabular}{|l|c|c|c|c|c|c|}
\hline Gamuts & \multicolumn{2}{|c|}{ Red Primaries } & \multicolumn{2}{c|}{ Green Primaries } & \multicolumn{2}{c|}{ Blue Primaries } \\
\hline & $\mathrm{x}$ & $\mathrm{y}$ & $\mathrm{x}$ & $\mathrm{y}$ & $\mathrm{x}$ & $\mathrm{y}$ \\
\hline Rec.709/sRGB & 0.640 & 0.330 & 0.300 & 0.600 & 0.150 & 0.060 \\
\hline DCI-P3 & 0.680 & 0.320 & 0.265 & 0.690 & 0.150 & 0.060 \\
\hline Projector & 0.680 & 0.320 & 0.265 & 0.690 & 0.140 & 0.070 \\
\hline Toast & 0.570 & 0.320 & 0.300 & 0.530 & 0.190 & 0.130 \\
\hline Toy & 0.510 & 0.320 & 0.310 & 0.480 & 0.230 & 0.190 \\
\hline
\end{tabular}

our approach are perceptually more faithful to the original image in terms of contrast and colors as compared with the results of Zamir et al. ${ }^{1}$ These simple modifications eliminate not only the problems with saturation and contrast in the reproduced images, but also the need to perform any sort of preprocessing as it was the case with the algorithm of Zamir et al. ${ }^{1}$

\section{EXPERIMENTS AND RESULTS}

In this section we present in detail the different stages of our framework, experimental setups, data collection and analysis.

\subsection{Methodology}

Our primary aim is to rank gamut extension algorithms according to their efficiency in cinema conditions using a digital cinema projector (Barco DP-1200 44 ). To this end we devise a framework, shown in figure 4, where given a wide gamut 


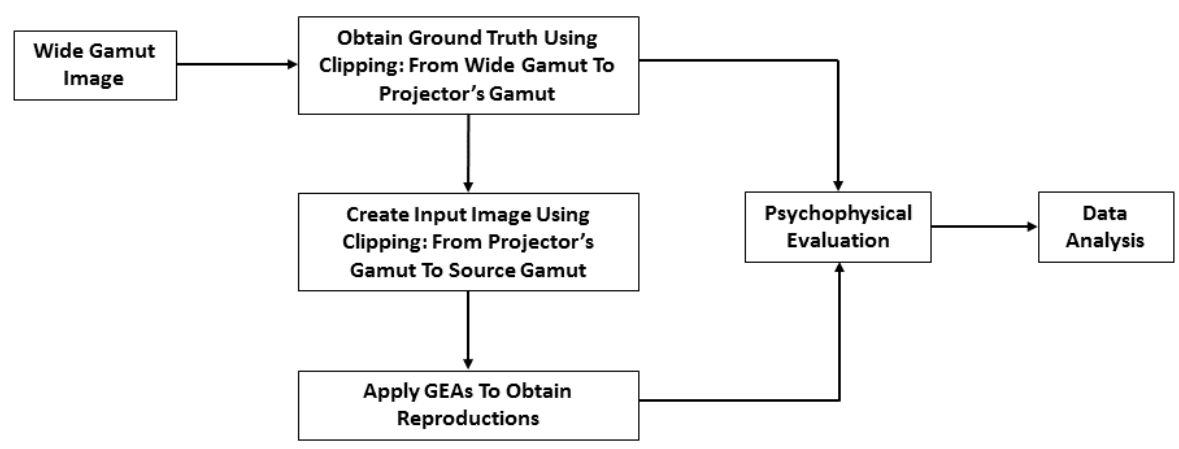

Figure 4: A schematic of our framework.

image we first create a ground truth by clipping (in xyY colorspace) those colors that fall outside the gamut of our cinema projector to the boundary of the projector's gamut. The gamut of our cinema projector has the same red and green primaries as in the cinema standard gamut DCI-P3, with a slightly different blue primaries. Nonetheless, both the DCI-P3 gamut and the projector gamut cover almost the same amount of area on the chromaticity diagram as it can be seen in figure 3 . Similarly, we create input images by applying a clipping operation on ground truth wide-gamut images to map out-of-gamut colors to the boundary of the Rec. 709 gamut (or any other desired source gamut). Some of the test images that we use in our experiments are shown in figure 5. Once we have the input images ready, we apply to them all five gamut extension algorithms to generate reproductions for three different experimental setups as we shall see in the following section. Next we perform subjective evaluation (for each experimental setup) using 17 observers; all of them were tested for normal color vision using the Ishihara color blindness test. We split the observers into two groups: inexperienced observers and experienced observers. The inexperienced observers don't have any formal training or experience with image processing and were never a part of any psychophysical experiment to judge images, whereas the experienced observers belong to image processing community and participated in various similar psychophysical experiments. We create low light ambience mimicking the lighting atmosphere of a real cinema. The illumination measured at the screen was around 750 lux and the ambient illuminance was 1 lux. During the experiment there wasn't any strong colored object in the field of view of observers. The glare-free screen used in our experiments is around 3 meter wide and has a height of 2 meter. Each observer was instructed to sit approximately 5 meters away from the screen. To avoid learning, we repeat the experiment on three different days for each observer. For each setup, observers are asked to choose (forced choice) which of two different gamut-mapped versions of an image is more faithful to the wide gamut ground truth. The observers were forced to pick one of the reproductions as we didn't include tie decision in our experiments. Finally, we analyze the psychophysical data using the work of Morovič, ${ }^{45}$ that is based on Thurstone's law of comparative judgement. ${ }^{46}$

\subsection{Creation of Wide Gamut Images}

To the best of our knowledge, there doesn't exist any standard dataset in the gamut mapping literature that can be used to evaluate GEAs. Therefore, we create images using a camera that is capable to capture RAW images: we associate wide gamut colorspace (ProPhoto RGB) with the RAW images to obtain true color images in Adobe Lightroom, and the rest of the process to obtain the ground truth and input images is just as explained in the previous section.

\subsection{Setups for Psychophysical Evaluation of GEAs}

We perform assessment of GEAs in the following experimental setups:

1. Mapping from a small gamut to the projector gamut: we map the source images from a small gamut, slightly smaller than the Rec. 709 gamut to the larger gamut of our cinema projector. We choose an input gamut smaller than Rec. 709 so as to have clear color differences in the reproductions, thus making the experimental task easier for observers.

The findings of psychophysical sessions are shown in figure 6a where it can be seen that the SDS algorithm produces images that are perceptually closer to the original material as compared with the other algorithms. The observers declared HCM as the least accurate algorithm, whereas both the CE and the LCA algorithms, due to the usage of the high chroma boost function described earlier, ranks third and fourth, respectively. Even though our proposed algorithm 

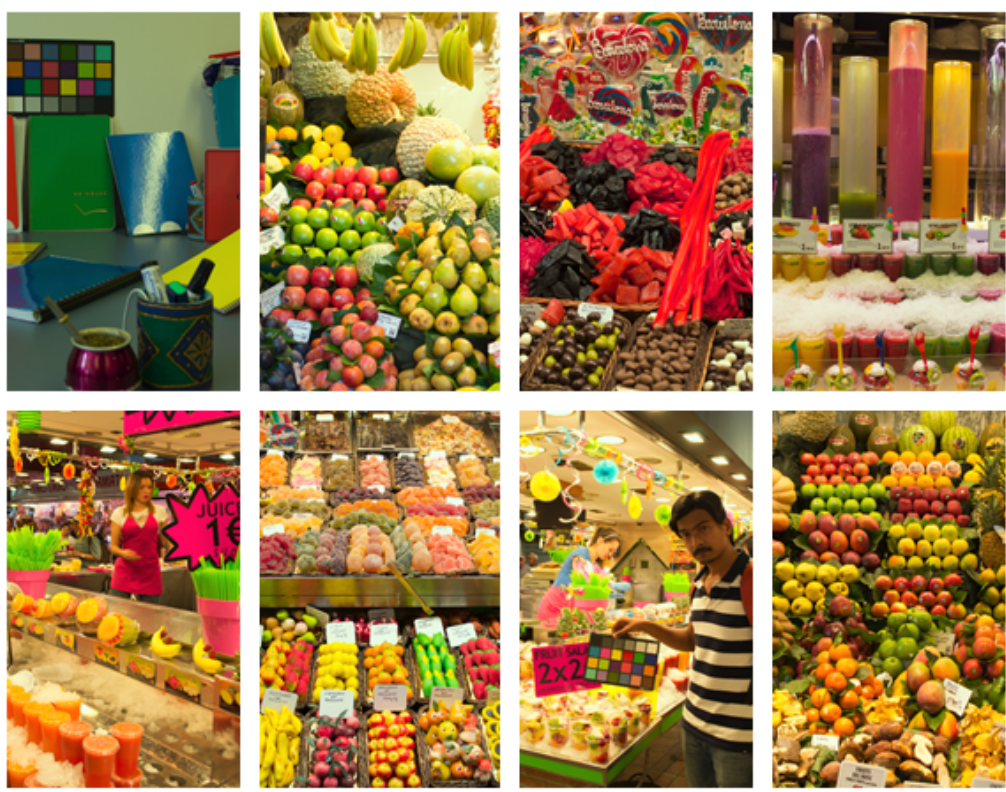

Figure 5: Test Images with Rec. 709 gamut.

ranks second, it provides a control that, instead of extending source colors to make full use of the target gamut, allows us to stop anywhere in between source and target gamuts. This is a useful property for any GE algorithm to exhibit in order to produce pleasant reproduction and avoid over-enhancement of memory colors, specially the skin tones which is always a key issue in movie postproduction.

2. Mapping from Rec. 709 to projector's gamut: in the previous setup we reported the efficiency of GEAs when there was a large difference between source and target gamuts, and also the input material was represented in a gamut smaller than the Rec. 709 gamut. However, in this setup we mimic the practical situation where the source material has Rec. 709 gamut and we map the source colors to the colors of the DCI-P3 gamut.

It can be seen in figure $6 \mathrm{~b}$ that the ranking order of GEAs is changed dramatically. The HCM algorithm that ranked as least accurate in the previous setup is now far more accurate algorithm as compared with the other competing algorithms. Trends for both of these setups show that the HCM algorithm reproduces gamut extended images accurately when the difference between source and destination gamut is small, whereas our algorithm, SDS, and CE generate reproductions more perceptually faithful when source and destination gamuts have fairly large color differences. And the LCA method produces poor gamut mapped images under both setup 1 and setup 2 .

3. Comparison only in some regions of interest: in the experimental setup 2, what we observed that many inexperienced observers had difficulty finding the differences between ground truth and reproductions. Therefore, to test the reliability of setup 2, we devise another setup where we ask color experts to mark some regions of interest and all observers perform the test considering image results inside those regions only. The results are reported in figure $6 \mathrm{c}$ where the trend is slightly changed for LCA and CE algorithms. Now the reproductions of LCA algorithm are ranked higher than the results of CE algorithm. The ranking trend for HCM, SDS and our algorithm is same as reported in the setup 2 .

\subsection{Objective metric suitable for GE Problem}

This particular section is devoted to identify an error metric that is suitable for the gamut extension problem. First, we examine the prediction quality of four error metrics: CIE $\Delta \mathrm{E}, \mathrm{CIE} \Delta \mathrm{E} 94,{ }^{37} \mathrm{CIE} \Delta \mathrm{E} 2000^{38}$ and CID. ${ }^{40}$ We test the efficiency of GEAs for setup 1 and setup 2 using these error metrics that find distortions between ground truth and reproductions. We show the overall error for all the images in setup 1 and setup 2 in figure 7 and figure 8 , respectively. 


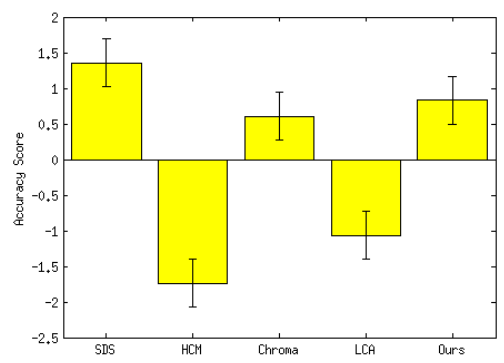

(a) Setup 1

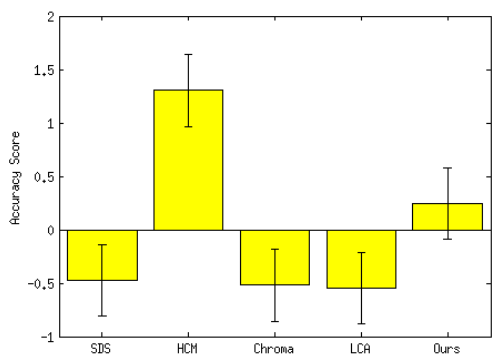

(b) Setup 2

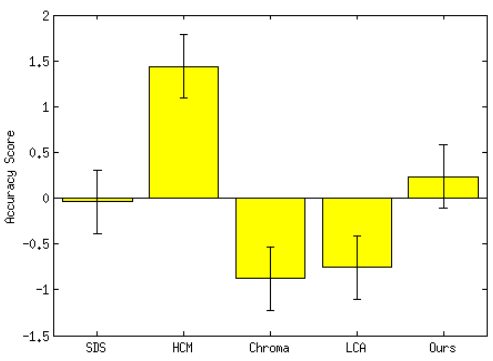

(c) Setup 3

Figure 6: Results of experiment, all images and observers.

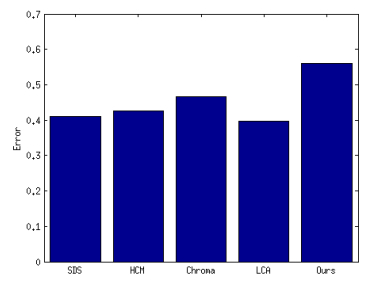

(a) CIE $\Delta \mathrm{E}$

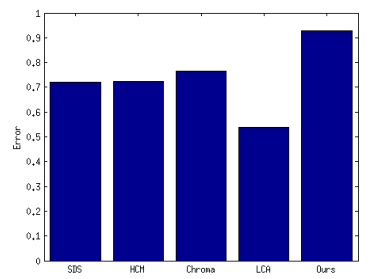

(b) CIE $\Delta$ E94

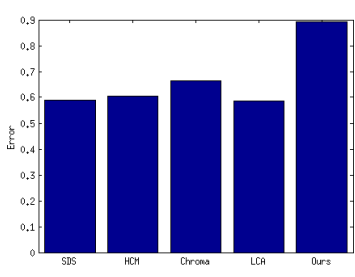

(c) CIE $\Delta$ E2000

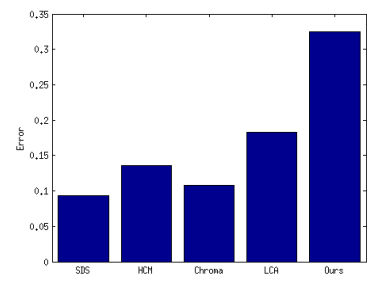

(d) CID

Figure 7: Predictions of error metrics in setup 1.

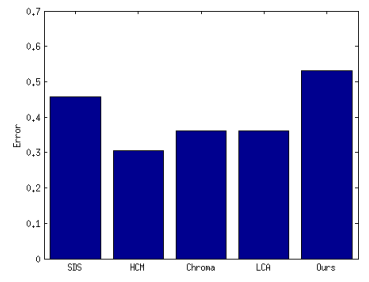

(a) CIE $\Delta \mathrm{E}$

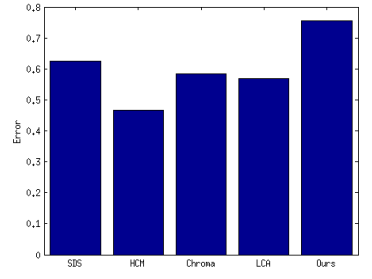

(b) CIE $\triangle$ E94

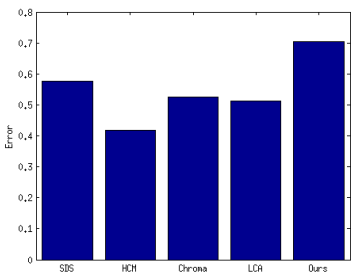

(c) CIE $\Delta$ E2000

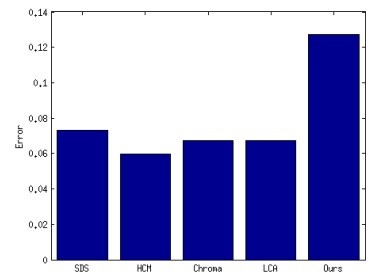

(d) CID

Figure 8: Predictions of error metrics in setup 2.

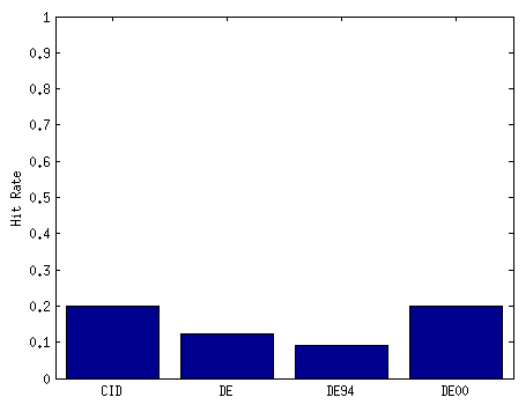

(a) Hit rate for setup 1

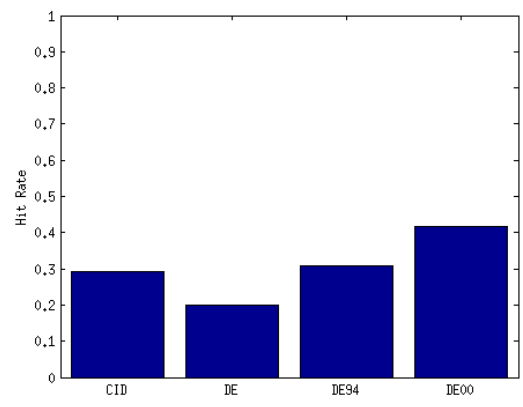

(b) Hit rate for setup 2

Figure 9: Hit rates obtained by image quality metrics for experimental setups.

Overall, all of these error metrics predict that the best performing method to map images from smaller source gamut to a larger destination gamut is the LCA algorithm, and it contradicts with the findings of psychophysical experiment in which 
the LCA algorithm ranks as the second least accurate algorithm. Another notable thing is that the trend of prediction for all three flavors of CIE $\Delta \mathrm{E}(\mathrm{CIE} \Delta \mathrm{E} 94$ and CIE $\Delta \mathrm{E} 2000)$ is almost similar for our dataset. To confirm the reliability of these image metrics, we check the correlation between the choices of our observers in the psychophysical experiment and the predictions of the error metrics. To achieve this, we use the hit rate $h$ approach that is defined as

$$
h=\frac{c}{N}
$$

where $N$ is the total number of choices for any experimental setup and $c$ is the number of correctly predicted choices.

Since our purpose is to find a metric that works for both small and large difference between source and target gamuts, in figure 9 we present the hit rates to validate the image quality metrics for both setup 1 and setup 2 . It is evident that none of these metrics achieves even half of the maximum theoretical hit rate of 1.0, meaning that the prediction accuracy is way below $50 \%$.

\section{CONCLUSION}

We presented a psychophysical study to evaluate our gamut extension algorithm and four other GEAs under cinematic conditions using a cinema projector. The analysis of psychovisual data showed that our algorithm and the SDS algorithm works efficiently when the difference between source and destination gamuts is large, whereas the HCM algorithm produces better images when the source-destination gamut pair has smaller differences in colors. Moreover, we tested the prediction quality of four error metrics in order to identify a suitable image measure for GE problem. We concluded that none of the test metrics have any useful correlation with the psychophysical choices made by the observers.

Even though our algorithm has a functionality to control the amount of colors extension using the threshold ( $\left.T_{e}\right)$, the reproductions are sometimes off (over-saturated, or under-saturated) from the ground truth for a few images. We leave it as a future work where we will make the threshold level $\left(T_{e}\right)$ adaptive according to the content of each image. Moreover, we notice that our algorithm causes hue shifts in some images and we intend to deal with this issue as well.

\section{ACKNOWLEDGEMENTS}

This work was supported by the European Research Council, Starting Grant ref. 306337, by Spanish grant ref. TIN201238112 and by ICREA Academia. The authors are grateful to all the participants for the psychophysical experiments. A special thank goes to Praveen Cyriac who helped us gathering the dataset and also running sessions of experiments. We would also like to thank Narciso García and Francisco Morán Burgos from Universidad Politécnica de Madrid and the Spanish section of MPEG for their help in providing us with access to test sequences. Last but not least, the authors would like to express their gratitude to Barco N.V. for their invaluable help, without which this work would not have been possible.

\section{REFERENCES}

[1] Zamir, S. W., Vazquez-Corral, J., and Bertalmío, M., "Gamut mapping in cinematography through perceptually-based contrast modification," IEEE Journal of Selected Topics in Signal Processing 8(3), 490-503 (2014).

[2] Morovič, J., [Color gamut mapping], vol. 10, Wiley (2008).

[3] Bertalmío, M., [Image Processing for Cinema], vol. 4, CRC Press, Taylor \& Francis (2014).

[4] http://spectrum.ieee.org/consumer-electronics/audiovideo/ lasers-coming-to-a-theater-near-you. [Online; accessed 29-July-2014].

[5] CIE, "Guidelines for the evaluation of gamut mapping algorithms," tech. rep., CIE 156 (2004).

[6] Sara, J. J., The automated reproduction of pictures with nonreproducible colors, PhD thesis, Massachusetts Institute of Technology (MIT) (1984).

[7] Marcu, G. and Abe, S., "Gamut mapping for color simulation on CRT devices," in [Proc. of Color Imaging: DeviceIndependent Color, Color Hard Copy, and Graphic Arts ], (1996).

[8] Johnson, A. J., "Perceptual requirements of digital picture processing," Paper presented at IARAIGAI symposium and printed in part in Printing World (1979).

[9] Herzog, P. G. and Müller, M., "Gamut mapping using an analytical color gamut representation," in [Proc. of Color Imaging: Device-Independent Color, Color Hard Copy, and Graphic Arts ], 117-128 (1997). 
[10] Meyer, J. and Barth, B., "Color gamut matching for hard copy," in [Proc. of SID Digest ], 86-89 (1989).

[11] Bala, R., Dequeiroz, R., Eschbach, R., and Wu, W., "Gamut mapping to preserve spatial luminance variations," Journal of Imaging Science and Technology 45, 122-128 (2001).

[12] Morovič, J. and Wang, Y., "A multi-resolution, full-colour spatial gamut mapping algorithm," in [Proc. of Color Imaging Conference], 282-287 (2003).

[13] Kimmel, R., Shaked, D., Elad, M., and Sobel, I., "Space-dependent color gamut mapping: A variational approach," IEEE Transactions on Image Processing 14, 796-803 (2005).

[14] McCann, J. J., "Lessons learned from mondrians applied to real images and color gamuts," in [Proc. of Color Imaging Conference ], 1-8 (1999).

[15] Land, E. H., "The retinex theory of color vision.," Scientific American 237(6), 108-128 (1977).

[16] Farup, I., Gatta, C., and Rizzi, A., "A multiscale framework for spatial gamut mapping," IEEE Transactions on Image Processing 16(10), 2423-2435 (2007).

[17] Zolliker, P. and Simon, K., "Retaining local image information in gamut mapping algorithms," IEEE Transactions on Image Processing 16(3), 664-672 (2007).

[18] Alsam, A. and Farup, I., "Spatial colour gamut mapping by orthogonal projection of gradients onto constant hue lines," in [Proc. of 8th International Symposium on Visual Computing], 556-565 (2012).

[19] Zamir, S. W., Vazquez-Corral, J., and Bertalmío, M., "Gamut mapping through perceptually-based contrast reduction," in [Proc. of the 6th Pacific-Rim Symposium on Image and Video Technology (PSIVT)], 1-11 (2013.).

[20] Bertalmío, M., Caselles, V., Provenzi, E., and Rizzi, A., "Perceptual color correction through variational techniques," IEEE Transactions on Image Processing 16(4), 1058-1072 (2007).

[21] Vazquez-Corral, J., Zamir, S. W., and Bertalmío, M., "Considering saliency in a perception inspired gamut reduction algorithm," in [Proc. of IS\&T/SID 22nd Color Imaging Conference (to be appeared)], (2014).

[22] Nakauchi, S., Hatanaka, S., and Usui, S., "Color gamut mapping based on a perceptual image difference measure," Color Research \& Application 24(4), 280-291 (1999).

[23] Hoshino, T., "A preferred color reproduction method for the HDTV digital still image system," in [Proc. of IS\&T Symposium on Electronic Photography], 27-32 (1991).

[24] Hoshino, T., "Color estimation method for expanding a color image for reproduction in a different color gamut," (May 1994). US Patent 5,317,426.

[25] Kang, B. H., Morovič, J., Luo, M. R., and Cho, M. S., "Gamut compression and extension algorithms based on observer experimental data," ETRI journal 25(3), 156-170 (2003).

[26] Anderson, H., Garcia, E., and Gupta, M., "Gamut expansion for video and image sets," in [Proc. of the 14th International Conference of Image Analysis and Processing - Workshops ], 188-191 (2007).

[27] Liu, Y., Song, G., and Li, H., "A hue-preserving gamut expansion algorithm in CIELUV color space for wide gamut displays," in [Proc. of the 3rd International Congress on Image and Signal Processing (CISP)], 2401-2404 (2010).

[28] Kim, M. C., Shin, Y. C., Song, Y. R., Lee, S. J., and Kim, I. D., "Wide gamut multi-primary display for HDTV," in [Proc. of 2nd European Conference on color Graphics, Imaging and Vision], 248-253 (2004).

[29] Casella, S. E., Heckaman, R. L., and Fairchild, M. D., "Mapping standard image content to wide-gamut displays," in [Sixteenth color imaging conference: color science and engineering systems, technologies, and applications], 106-111 (2008).

[30] Muijs, R., Laird, J., Kuang, J., and Swinkels, S., "Subjective evaluation of gamut extension methods for wide-gamut displays.," in [Proc. of the 13th International Display Workshop], 1429-1432 (2006).

[31] Laird, J., Muijs, R., and Kuang, J., "Development and evaluation of gamut extension algorithms," Color Research \& Application 34(6), 443-451 (2009).

[32] Heckaman, R. L. and Sullivan, J., "18.3: Rendering digital cinema and broadcast TV content to wide gamut display media," SID Symposium Digest of Technical Papers 42(1), 225-228 (2011).

[33] Li, Y., Song, G., and Li, H., "A multilevel gamut extension method for wide gamut displays," in [Proc. of International Conference on Electric Information and Control Engineering (ICEICE), ], 1035-1038 (2011).

[34] Dugay, F., Farup, I., and Hardeberg, J. Y., "Perceptual evaluation of color gamut mapping algorithms," Color Research \& Application 33(6), 470-476 (2008).

[35] Montag, E. D. and Fairchild, M. D., "Psychophysical evaluation of gamut mapping techniques using simple rendered images and artificial gamut boundaries," Image Processing, IEEE Transactions on 6(7), 977-989 (1997). 
[36] Bonnier, N., Schmitt, F., Brettel, H., and Berche, S., "Evaluation of spatial gamut mapping algorithms," in [Proc. of IS\&T/SID 14th Color Imaging Conference ], 56-61 (2006).

[37] Berns, R. S., "The mathematical development of CIE TC 1-29 proposed colour difference equation: CIELCH," in [Proc. of the Seventh Congress of International Colour Association, B, C19.1C19.4], (1993).

[38] Luo, M. R., Cui, G., and Rigg, B., "The development of the CIE 2000 colour-difference formula: CIEDE2000," Color Research \& Application 26(5), 340-350 (2001).

[39] Barańczuk, Z., Zolliker, P., and Giesen, J., "Image quality measures for evaluating gamut mapping," Color and Imaging Conference 2009(1), 21-26 (2009).

[40] Lissner, I., Preiss, J., Urban, P., Lichtenauer, M. S., and Zolliker, P., "Image-difference prediction: From grayscale to color.," IEEE Transactions on Image Processing 22(2), 435-446 (2013).

[41] Hardeberg, J. Y., Bando, E., and Pedersen, M., "Evaluating colour image difference metrics for gamut-mapped images," Coloration Technology 124(4), 243-253 (2008).

[42] Rizzi, A., Gatta, C., and Marini, D., "A new algorithm for unsupervised global and local color correction," Pattern Recognition Letters 24, 1663-1677 (2003).

[43] Bertalmío, M., Caselles, V., and Provenzi, E., "Issues about retinex theory and contrast enhancement," International Journal of Computer Vision 83(1), 101-119 (2009).

[44] http://www.barco.com/en/Products-Solutions/Projectors/Digital-cinema-projectors/ 2K-Digital-cinema-projector-for-screens-up-to-12m-40ft.aspx. [Online; accessed 9-January-2015].

[45] Morovič, J., To Develop a Universal Gamut Mapping Algorithm, PhD thesis, University of Derby, UK (1998).

[46] Thurstone, L. L., “A law of comparative judgment," Psychological Review 34(4), 273-286 (1927). 